\title{
A Visual Navigation Control Method of Substation Inspection Robot
}

\author{
Liqiang $\mathrm{ZHONG}^{1 *}$, Shaosheng FAN ${ }^{2}$, Shaohai ZHANG ${ }^{2}$, Di YANG \\ ${ }^{1}$ Electric Power Research Institute of Guangdong Power Grid Co., Ltd. Guangzhou, Guangdong 510080, China \\ Email: liqiangzhong@hotmail.com \\ ${ }^{2}$ College of Electric and Information Engineering Changsha University of Science and Technology, Changsha, China \\ Email: fss508@163.com \\ +* Corresponding author
}

\begin{abstract}
According to the real condition of the substation inspection robot provided a vision-based navigation control method for substation inspection robot can be run in the complex road environment, with strong anti-interference, implementation of simple, good stability and high precision in this paper. In the rational planning of substation robot inspection path, making robots able to inspect substation equipment fully. Robots get information of navigation path through the visual, using HSV color space model effectively solve the interference of illumination in the image, then calculate the current offset of the center, and sent it to the controller, adjusting left and right wheels with differential speed make the robot running stably.
\end{abstract}

Keywords-substation inspection robot; navigation path; rebuild gray image; template matching

\section{INTRODUCTION}

With the development of automation technology, robot techniques are widely used in substation. There are lots of navigation methods for inspection robot, such as magnetic track navigation, inertial navigation, GPS navigation, visual navigation etc. The magnetic track navigation method is simple, reliable and has high navigation accuracy, but this method has to change the layout of substation by laying magnetic track for navigation which is inconvenient for lots of stations to build extra equipment[1-2]. The inertial navigation method needs to install the wireless device for signal transmitting and receiving in the work environment, it is easy to install, but the decentralized wireless device provides poor stability. The GPS navigation method will not change the layout of the station, but it is inaccurate and cannot meet the security requirements of the substation inspection.

Visual navigation method overcomes the shortcomings mentioned above; it is simple, reliable, accurate and easy to install [3]. However, the visual navigation method highly depends on the lighting conditions, inaccurate recognition of the navigation path will be caused by complex environments such as strong sunlight, shadow, rainy days etc. [4-5].

In order to improve the performance of visual navigation in complex environments, a robust visual navigation method for substation inspection robot is proposed in this paper [6]. Utilizing the robust characteristic to light changes in HSV color model, the proposed method can extrude the navigation path well in complex environments, and the template matching method and Outs method are applied to improve the accuracy in path recognition [7]. And a few more steps which are needed in dark environments and situation of 'Out of the path' are designed.

\section{NAVIGATION IN COMPLEX ENVIRONMENTS}

Considering various complex conditions such as strong sunlight, shadows, rainy days etc. the proposed navigation method decompose the image of the navigation path into $\mathrm{H}$ (hue), $\mathrm{S}$ (saturation), $\mathrm{V}$ (value) components, and reconstruct the image by reunion these three components in different weights to extrude the navigation path. Then apply the template matching process to find whether the frame of image is acceptable and extract the navigation path using Otsu theory. And at last, the calculation for navigation can be implemented.

\section{A. Recognition of Navigation Path}

\section{1) Color space transformed}

Color image is constituted by red component, green component and blue component that are R, G, and B three components. HSV color space has its unique advantages in image processing. First, the $\mathrm{H}$ component and the $\mathrm{V}$ component are independent and separate, and the $\mathrm{H}$ component has nothing to do with color information of image. Secondly, the H component and the S component are independent, and will not change with the light intensity, which makes the HSV model is very appropriate for image algorithm processing. In order to better eliminate disturbance of the complex environmental to image, the color image must first be transformed into the HSV color space [8].

In image the $\mathrm{R}$ component value of each pixel is $\mathrm{R}(i, j)$, and the effective value of it is $[0,255]$. The $G$ component value of each pixel is $G(i, j)$, and the effective value of it is $[0,255]$. The B component value of each pixel is B $(i, j)$, and the effective value of it is $[0,255]$. Then we define the max $(i$, $j$ ) as the maximum value and $\min (i, j)$ as the minimum value of the R, G, B three components value of each pixel $(i, j)$.

$$
\begin{aligned}
& \max (i, j)=\operatorname{MAX}(R(i, j), G(i, j), B(i, j)) \\
& \min (i, j)=\operatorname{MIN}(R(i, j), G(i, j), B(i, j))
\end{aligned}
$$


The Value (V) component represents color brightness, shown on fig. $1(\mathrm{~d}), \mathrm{V}(i, j)$ is obtained as follows:

$$
V(i, j)=\max (i, j)
$$

The Saturation (S) represents color purity, shown on fig. $1(\mathrm{c}), \mathrm{S}(i, j)$ is obtained as follows:

$$
S(i, j)= \begin{cases}\frac{V(i, j)-\min (i, j)}{V(i, j)} & V(i, j) \neq 0 \\ 0 & V(i, j)=0\end{cases}
$$

The value of Hue $(\mathrm{H})$ is a symbol of difference between different colors, shown on fig.1 (b), for each pixel in an image $\mathrm{H}(i, j)$ can be calculated by the following formula:

$$
H(i, j)=\left\{\begin{array}{lll}
\frac{60(G(i, j)-B(i, j))}{V(i, j)-\min (i, j)} & \text { IF } & V(i, j)=G(i, j) \\
120+\frac{60(B(i, j)-R(i, j))}{V(i, j)-\min (i, j)} & \text { IF } & V(i, j)=B(i, j) \\
240+\frac{60(R(i, j)-G(i, j))}{V(i, j)-\min (i, j)} & \text { IF } & V(i, j)=R(i, j)
\end{array}\right.
$$

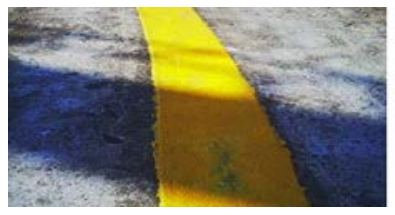

(a)

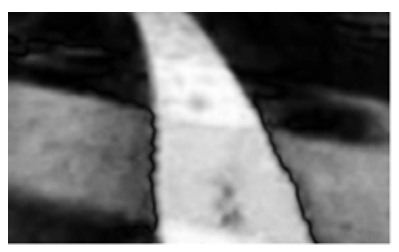

(c)

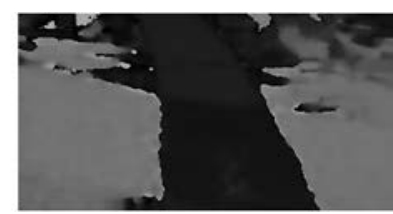

(b)

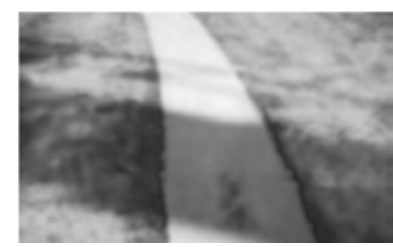

(d)
Figure 1. An original image of navigation path and its each HSV component. (a) the original image. (b) H component. (c) S component. (d) V component.

\section{2) Rebuild gray image}

Each component in HSV color model has different features, to reduce the influence caused by lights changes, image $\mathrm{F}$ should be reconstructed by reunion the $\mathrm{H}, \mathrm{S}, \mathrm{V}$ components in different weights. From fig.1 (b) and fig.1 (d) we can see that the characteristics of navigation path are basically opposite showing on the $\mathrm{H}$ component and $\mathrm{V}$ component. According to this feature we can use normalization method to enhance and extraction preliminary characteristics information of navigation path. First of all, we convert the $\mathrm{H}$ component into the inverse image:

$$
\bar{H}(i, j)=255-H(i, j)
$$

Secondly, normalizing the inverse image $\bar{H}$, here the range of normalization is not $[0,1]$, where $\mathrm{a}$ is the maximum value of $\bar{H}$ and $\mathrm{b}$ is the minimum value of $\bar{H}$. Then we have the coefficient matrix $H^{\prime}$ and the effective value of it is between $\mathrm{a} / 255$ to $\mathrm{b} / 255$ :

$$
H^{\prime}(i, j)=\bar{H}(i, j) \times \frac{(b-a)}{255^{2}}+\frac{a}{255}
$$

This avoids the image in the process of normalization is forced to zoom in and out. When we reconstruct the gray image the $\mathrm{S}$ component is added to the $\mathrm{V}$ component and then multiplied by the coefficient matrix:

$$
F(i, j)=(2 \times S(i, j)+V(i, j)) \times H^{\prime}(i, j)
$$

In order to eliminate interference of the shadow in the image efficiently, we can increase the weight of the $\mathrm{S}$ component in the process of gray image reconstruction. Here, we choose to double the $\mathrm{S}$ component and the result of rebuild gray image. In this way, to make the navigation path prominent we can construct the gray image without the interference from the strong illumination, shadows, surface water, and other outside objects.

3) Template Matching

To improve the accuracy in navigation, template matching is applied to match two images that contain common visual information and find the location of template image within the reference image. Here, the reference image is the rebuild gray image, the aim of the template matching is that found the key characteristics information of the navigation path of the template image in the rebuild gray image to ensure inspection robot running in the navigation path. The Normalized Cross-Correlation (NCC) method is one of the successful template matching methods and it is widely used in image matching application. Suppose I $(\mathrm{i}, \mathrm{j})$ is the reference image of size $\mathrm{M} * \mathrm{M}$ pixel and $\mathrm{T}(\mathrm{i}-\mathrm{u}, \mathrm{j}-\mathrm{v}$ ) is the template image of size $\mathrm{N} * \mathrm{~N}$ pixel. Fig. 2(a) shows the template image and fig. 2(b) is the result of template matching.

$$
\mu(u, v)=\frac{\sum_{i=0}^{N-1} \sum_{j=0}^{N-1} I(i, j) T(i-u, j-v)}{\sqrt{\sum_{i=0}^{N-1} \sum_{j=0}^{N-1} I^{2}(i, j) \sum_{i=0}^{N-1} \sum_{j=0}^{N-1} T^{2}(i-u, j-v)}}
$$

Where $\mathrm{u}, \mathrm{v}=0,1,2 \ldots \mathrm{M}-\mathrm{N}$. The largest value of $\mu$ is the best match between the rebuild gray and template image. In general, the NCC value ranges from 0 to 1 . The perfect 
matching between the rebuild gray image $F(i, j)$ and template image $T(i-u, j-v)$ is completed when the maximum value of NCC $\mu=1$ or $1-\mu=0$. To find the maximum value of $\mu$, and if the $\mu$ is larger than the predetermined threshold value, then matching is success; otherwise, the matching is unsuccessful, robot ending the current flow of images, grabbing the next frame image again. Here, the matching threshold is $\mu_{0}=0.85$.

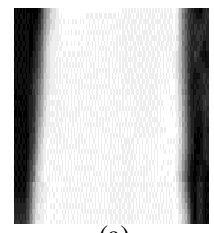

(a)

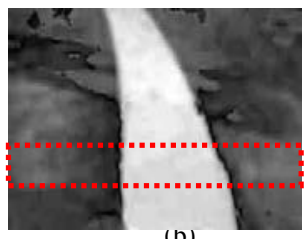

(D)

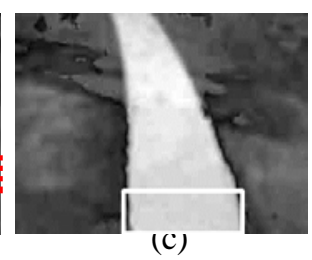

(c)
Figure 2. Template matching. (a) the template image T. (b) the area to be matched. (c) the result of template matching

4) Thresholding segmentation with otsu (maximizing inter-class variance)

The stationary threshold for segmentation will cause error in complex and changeable environment in image, so we adopt the thresholding segmentation with Otsu is utilized to extrude the navigation path. Otsu method is an adaptive threshold segmentation method; it can perform automatically the clustering-based image thresholding or the reduction of a gray-level image to a binary image. We define a twodimensional image of $F^{\prime}$ as $[f(x, y)]_{m \times n}$, where $\mathrm{m}$ and $\mathrm{n}$ represent the width and height of image, where $f(x, y)$ is the gray values at point $(x, y)$. Define the counts of gray level as $\mathrm{L}$, and $f(x, y) \in\{0,1, \ldots L-1\}, k_{i}$ is the number of gray level $\mathrm{i}$ in image, and $p_{i}$ is the probability of $k_{i}$, then we have:

$$
p_{i}=\frac{k_{i}}{m \times n}, \quad \text { and } \quad p_{i}>0, \sum_{i=o}^{L-1} p_{i}=1
$$

$S_{1}$ and $S_{2}$ are the foreground and background class of the image respectively, separated by gray level $t$, then the probability of $S_{1}$ and $S_{2}$ :

$$
p_{1}=\sum_{i=0}^{t} p_{i}, p_{2}=\sum_{i=t+1}^{L-1} p_{i}
$$

The center for classes $S_{1}$ and $S_{2}$ are $\omega_{1}, \omega_{2}$ respectively:

$$
\omega_{1}=\sum_{i=0}^{t} i p_{i} / p_{1}, \omega_{2}=\sum_{i=t+1}^{L-1} i p_{i} / p_{2}
$$

Minimizing the inter-class variance is the same as maximizing intra-class variance $\sigma^{2}$ :

$$
\sigma^{2}=p_{1}\left(\omega_{1}-\omega_{2}\right)^{2}-p_{2}\left(\omega_{1}-\omega_{2}\right)^{2}
$$

Desired threshold corresponds to the maximum $\tilde{\sigma}^{2}$ :

$$
\tilde{\sigma}^{2}=\operatorname{MAX}\left(\sigma^{2}(t)\right)
$$

Only the gray-level threshold $\mathrm{t}$ is function of $p_{1}, p_{2}, \omega_{1}$ and $\omega_{2}$, there is always a threshold $t_{0}$ for $\sigma^{2}\left(t_{0}\right)=\tilde{\sigma}^{2}$, making each frame to achieve the best results. Then we have the binary image $F$ :

$$
F= \begin{cases}0 & f(x, y)<t_{0} \\ 255 & f(x, y) \geq t_{0}\end{cases}
$$

Fig.3 is two conditions result of thresholding segmentation with Otsu, fig.3 (e) is the result of the strong light intensity, and fig.3 (f) is the results of shadows. The navigation path and the road can segment well under the different environment. But the segmentation threshold $t_{0}$ are different, fig.3 (e) is 133 and fig.3 (f) is 127.

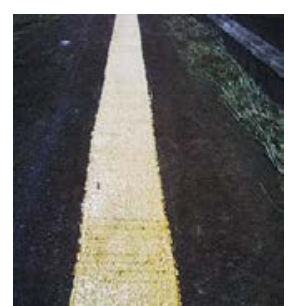

(a)

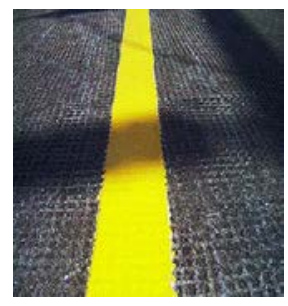

(d)

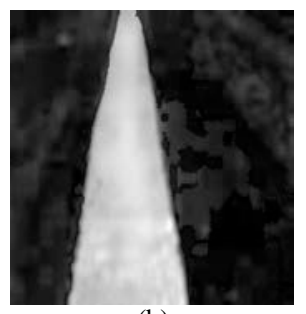

(b)

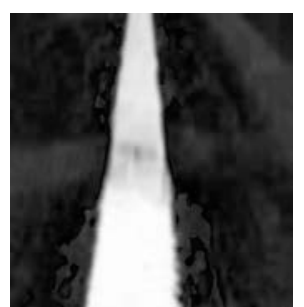

(e)
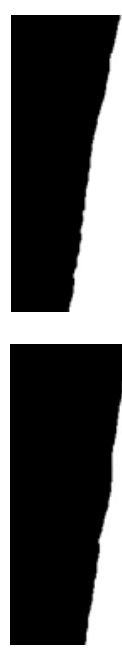

(C)
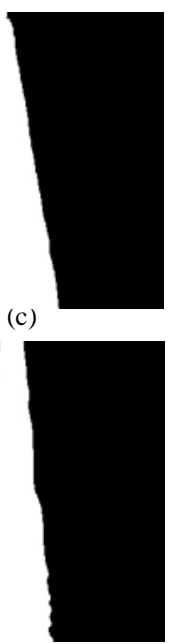

(f)
Figure 3. Two conditions thresholding segmentation with Otsu. (a) the original image with strong light intensity. (b) the rebuild gray image with shadows. (c) Otsu thresholding segmentation with strong light intensity. (d) the original image with shadows. (e) the rebuild gray image with strong

light intensity. (f) Otsu thresholding segmentation with shadows.

\section{B. Calculation of Navigation}

The information used for navigation needs to be calculated based on the navigation path extruded before. The

image size is $\mathrm{m} * \mathrm{n}$ and the center is $C_{0}\left(\frac{m}{2}, \frac{n}{2}\right)$, supposed $\Delta_{0}$ is $\mathrm{X}$-direction offset of navigation path form the center. According to (14) in some extreme circumstances there may be some noise in binary image, then we first calculate the average of $\mathrm{X}$ coordinate all the point each row which the pixel values is not equal to zero, $\bar{X}_{j}$ : 


$$
\bar{X}_{j}=\sum_{i=0}^{m} x_{i} / k, \quad f\left(x_{i}, j\right) \neq 0, j=0,1,2, \ldots, n
$$

Where $\mathrm{j}$ is the Y-coordinate, represents each row in the image, and $x_{i}$ is $\mathrm{X}$-coordinate of the pixel values is not equal to zero, and $\mathrm{k}$ is the counts of $X_{i}$ each row. According to (16) we have all rows average of $\mathrm{X}$ coordinate $\bar{X}_{j}$, and use that to fit the center line $L_{1}$ with least square, then we have L's gradient $\theta$. The navigation path in the upper part of image can reflect current trends in extension of the navigation path better; therefore, when calculating the offset process for each row we use the weighted average method. Here, according to experiments, each row weighting of coefficient $\alpha_{j}$ :

$$
\alpha_{j}=-\frac{1}{600} \times j+1.2 \quad j=0,1,2, \ldots, n
$$

Then, we have the weighted average of the offset $\Delta_{0}$ :

$$
\Delta_{0}=\sum_{j=0}^{n} \alpha_{j} \frac{\bar{X}_{j}-m / 2}{n}
$$

Finally, the extension direction of fitting line L reflected the movement trends of inspection robot, so the ultimate offset is not $\Delta_{0}$,but $\Delta$, that the difference between the $\mathrm{X}$ coordinate of $\mathrm{X}$ axis and L's node $C_{2}\left(x_{2}, 0\right)$ and half the width of the image $(\mathrm{m} / 2)$. Taking into account the offset $\Delta$ will not only reflect the size of robot offset, but also reflect the direction of the offset that if $\Delta<0$ the offset the direction is left and if $\Delta>0$ the offset the direction is right, fig.4(c) shown the calculation schematic graph, and $\Delta$ was calculated using:

$$
\Delta=\left\{\begin{array}{ll}
\Delta_{0}-\frac{n}{2 \tan \theta} & \theta \neq \frac{\pi}{2} \\
\Delta_{0} & \theta=\frac{\pi}{2}
\end{array} \quad, \quad-\frac{m}{2} \leq \Delta \leq \frac{m}{2}\right.
$$

Fig.3 (a) and fig.3 (d) are considered as the original images, and the size is $320 * 240$. According to the calculation method of section 2.4, fig.4 (b), (c) show the calculation results of that three navigation images. The red part is the recognized navigation path, the yellow line is the center line $\mathrm{L}$ of navigation path, and the yellow small circle is the center of navigation path. From fig. 4 and table I, it is observed that the calculation results of navigation provided the useful navigation information for robot that can reflect the robot running tendency perfectly.

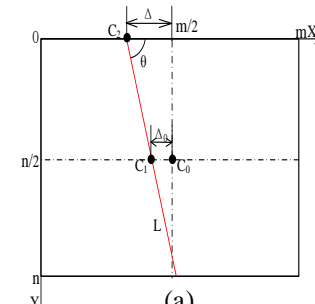

(a)

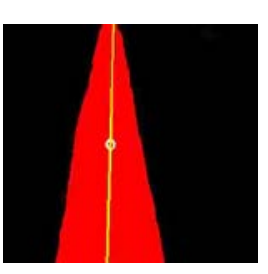

(b)

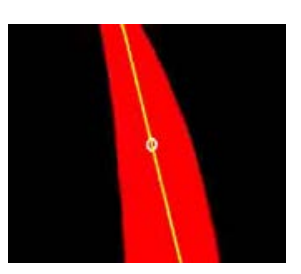

(c)
Figure 4. Calculation results of navigation. (a) the calculation schematic drawing. (b) the calculation result of fig.3(a). (c) the calculation result of fig.3 (d).

TABLE I. CALCUlATION RESUlts OF FIG.4 (B), (C), AND THE IMAGE SIZE IS $320 * 240$

\begin{tabular}{|c|c|c|c|}
\hline Figure & $\begin{array}{c}\text { Gradient } \\
(\boldsymbol{\theta})\end{array}$ & $\begin{array}{c}\text { Center } \text { offset } \\
\left(\Delta_{0}\right)\end{array}$ & $\begin{array}{c}\text { Offset } \\
(\Delta)\end{array}$ \\
\hline fig. 4(b) & $0.417 \pi$ & 5.277 & -27.005 \\
\hline fig. 4(c) & $-0.482 \pi$ & -18.920 & -13.055 \\
\hline
\end{tabular}

\section{Navigation Control Method in Curve}

This paper presents a navigation path detection method based on a straight-curve. The method has the advantages of easy operation and high stability when dealing with the linear navigation path and given the right offset in the curve navigation path, to ensure that the robot in the curve smooth walking. Robot in the linear navigation path, according to the results of the navigation image processing analysis of the current possibility of the curve, if the robot enters the curve, then fit the curve.

From the first line of the navigation image began to search down to find the existence of a point $P_{3}$, that the above region of $P_{3}$ point does not exist navigation path and the below region exist the navigation path. If you can find point $P_{3}$, then this is the curve navigation path, otherwise, where the linear navigation path. Fig. 5 shows the process of curve navigation path judgment, fitting and calculation and Fig.6 shows the change of offset when the robot turning.
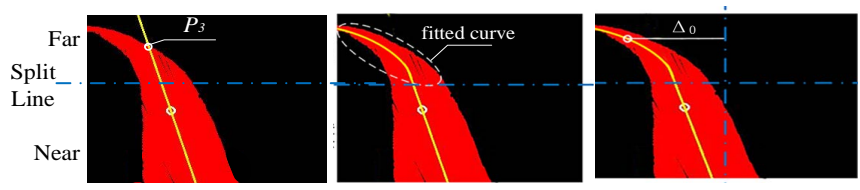

Figure 5. The process of curve navigation path judgment, fitting and calculation.

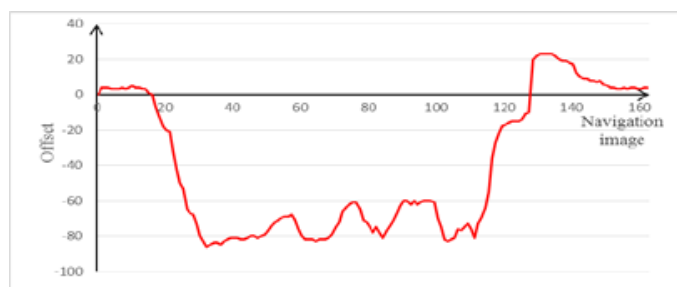

Figure 6. The change of offset when the robot turning 


\section{Horizontal Angle Control Method of Supplementary Light}

In order to make the robot can carry out inspection work in the evening and ensure continuity and stability of the inspection, it is necessary to install a light source directly on the front of the robot and follow the navigation path in front of it so that robots can be inspected even in darker nights. After entering the curve, in front of the robot on the road to form a bright area, the formation of strong interference in the navigation image processing. Therefore, in order to solve this problem, so that the robot at night in the curve can be more stable operation, the need to adjust the direction of the light to ensure that has been directed to the navigation path, that is, the direction of light irradiation is controllable, and corresponds with the navigation path bending angle. Fig.7 shows the schematic illustration of horizontal angle of supplementary light when the robot turning.
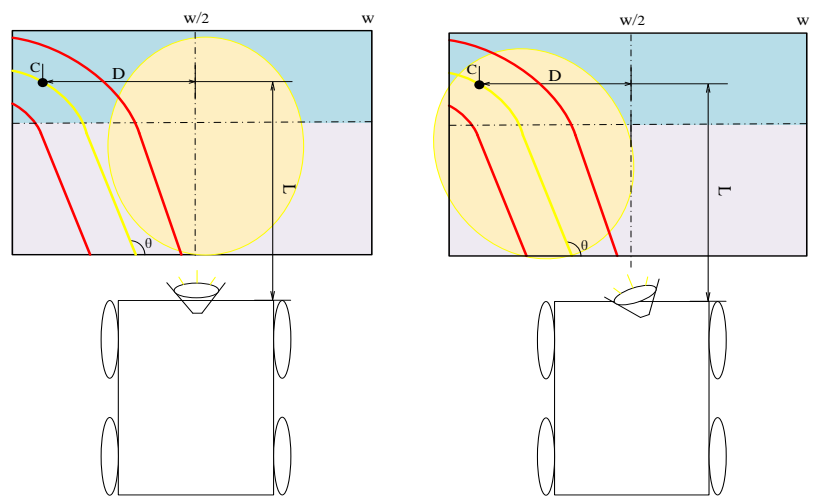

Figure 7. Horizontal angle of supplementary light when the robot turning

This paper presents a control method that the horizontal angle of the lamp can be adjusted with the offset of the image. Since the navigation image is the image of the navigation path in front of the inspection robot, the offset always reflects the movement tendency of the inspection robot. When the horizontal angle of the fill light on the inspection robot is adjusted as the offset amount changes, the direction of the light will always follow the geometric shape of the curve changes, into a dynamic adjustment mode. Set the horizontal angle of the light is $\varphi$, light irradiation area is relatively close, do not need to adjust precisely so that the horizontal adjustment angle of the light can be analyzed from the image plane:

$$
\varphi=\arctan (D / L)
$$

The $\mathrm{D}$ can be deduced from the image offset, $D=k \Delta_{0}, \mathrm{k}$ is the scale coefficient, unit: $\mathrm{cm}$ per pixel. The 15-centimeter navigation mark line is 60 pixels in the middle of the image, so $\mathrm{k}$ takes $0.25 \mathrm{~cm}$ per pixel. $\mathrm{L}$ is the distance between the front end of the inspection robot and the actual position corresponding to the image center. According to the actual measurement, it is $120 \mathrm{~cm}$, then

$$
\varphi=\arctan \frac{0.25 \Delta_{0}}{120} \approx \arctan \left(0.002 \Delta_{0}\right)(\text { rad })
$$

In the actual operation process, because the inspection robot running speed is relatively slow, fill light lamp irradiation area is large enough, so fill light horizontal deflection angle only in a certain interval to adjust. According to (21) and a large number of experiments, the image offset of the inspection robot corresponds to the horizontal angle of the supplementary light, and the correspondence relationship is shown in Table II(image size is $\left.320 \times 240, \Delta_{0}>0\right)$.

TABLE II. IMAGE OFFSET AND HORIZONTAL ANGLE OF SUPPLEMENTARY LIGHT CORRESPONDENCE TABLE

\begin{tabular}{|c|c|}
\hline Image Offset $\Delta_{0}$ ( pixel) & $\begin{array}{c}\text { Horizontal Angle of } \\
\text { Supplementary Light } \boldsymbol{\varphi}\left({ }^{\circ}\right)\end{array}$ \\
\hline $0 \leq \Delta_{0}<30$ & 0 \\
\hline $30 \leq \Delta_{0}<50$ & 4 \\
\hline $50 \leq \Delta_{0}<70$ & 6 \\
\hline $70 \leq \Delta_{0}<90$ & 8 \\
\hline $90 \leq \Delta_{0}<110$ & 11 \\
\hline $110 \leq \Delta_{0}<130$ & 14 \\
\hline$\Delta_{0} \geq 130$ & 19 \\
\hline
\end{tabular}

\section{E. Navigation in Dark Environment}

In order to overcome the shortage of the ordinary lights in dark environment, this paper proposes a brightness detection method based on $\mathrm{V}$ component and $\mathrm{S}$ component of the HSV image. In the HSV color space, to the V component, the mean of brightness $V_{p}$ was given as

$$
V_{p}=\sum_{i=0}^{255} i \times c(i) / \sum_{i=0}^{255} c(i)
$$

Where $i$ is the brightness value in $\mathrm{V}$ component and c ( $i$ ) is the pixel count in brightness $i$. At the same time, the $\mathrm{S}$ component, the mean of saturation $S_{p}$ was given as

$$
S_{p}=\sum_{i=0}^{255} i \times s(i) / \sum_{i=0}^{255} s(i)
$$

Where $i$ is the saturation value in $\mathrm{S}$ component and $\mathrm{s}(i)$ is the pixel count in saturation $i$.

Then, based on the equation $22 \sim 23$, judging the image is the too bright or too bright with results of the criteria 1 and criteria 2 . The criteria 1 is as follows:

$$
V_{p}>150 \& \& S_{p}<80
$$

The criteria 2 is as follows:

$$
V_{p}<75 \& \& S_{p}>60
$$


Robot will adjust intensity of the supplementary lighting with the result of the judgment about brightness information which in the current image. So that the navigation path on the different road sections have the same light intensity and guarantee the consistency and stability of visual images ${ }^{\text {[9] }}$.

\section{F. Out of the Navigation Path and Automatic Return}

An automatic return method should be applied when inspection robot run out of the path.

\section{1) Conditions of judgement}

Even if out of the navigation path in some special cases, robot can automatically query the historical data to determine the current direction of the out of the navigation path, and then adjust in the opposite direction back to the navigation path.

- Condition1: Jumping of the Otsu threshold. When robots walk on the navigation path, the Otsu threshold is relatively stable, while the robot through the contrasts of illumination or deviate from the navigation path Otsu the threshold would make abrupt change.

- Condition2: Results of the template matching. According to the results of the template matching if the matching is unsuccessful robot may have been out of the navigation path.

- Condition3: Offset trends. When robot walking on the navigation path, the offset are relatively stable, too. According to historical data we can know the trends of offset when the offset mutations and presents irregular changes.

If all of the above conditions (Condition 1 to 3 ) are satisfied, then the robot was out of the navigation path already.

\section{2) Processing method}

When the robot out of the navigation path, first of all, stop immediately and alert information to the controller enables the robot to enter adjust status; Secondly, according to the continuous changes of L's gradient $\theta$ and the value of offset $\Delta_{0}$ to determine the direction of the out of navigation path, that if $\Delta_{0}<0$, robot is turn left and if $\Delta_{0}>0$ robot is turn right; Thirdly, by differential adjusting speed of the left and right wheels make the robot turn back to the navigation path.

\section{EXPERIMENTS AND APPLICATIONS}

In this section, we test the proposed method in experiments and applied it to the practical application.

\section{A. Experiments}

We conduct the experiments in different situations and it is detailed in the following.

\section{1) Experiment of dark environment}

To make robot running in the dark environment well, the suitable supplemental is important. Fig.8 (a) is the condition that supplemental lighting is too bright, fig.8 (b) is the condition that supplemental lighting is too dark, while fig.8 (c) is the suitable supplemental lighting intensity. According to table III the result of the judgment about brightness information, fig.8 (a) satisfied the criteria 1and fig.8 (b) satisfied the criteria 2 .

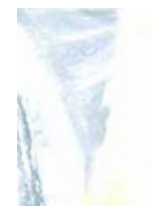

(a)

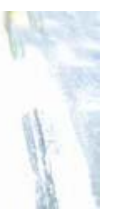

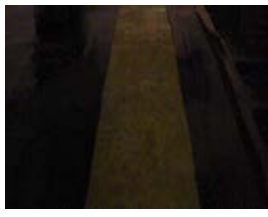

(b)

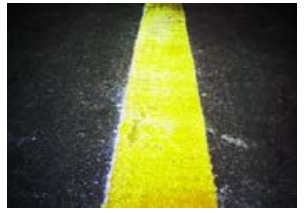

(c)
Figure 8. Inspecting in evening. (a) the supplemental lighting is too bright. (b) the supplemental lighting is too dark. (c) the suitable supplemental lighting intensity

TABLE III. THE RESULT OF JUDGMENT FIG.8 (A), (B), (C)

\begin{tabular}{|c|c|c|c|}
\hline Figure & $V_{p}$ & $S_{p}$ & Result \\
\hline fig.8(a) & 193.96 & 10.7 & Bright \\
\hline fig.8(b) & 55.21 & 81.63 & Dark \\
\hline fig.8(c) & 93.19 & 66,57 & Suitable \\
\hline
\end{tabular}

2) Experiment of out the navigation path and automatic return

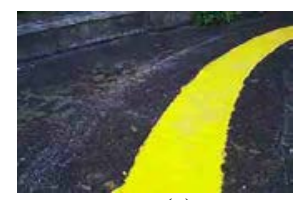

(a)

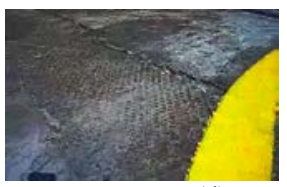

(d)

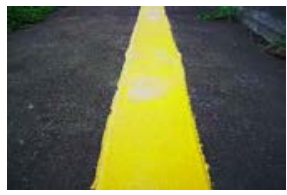

(b)

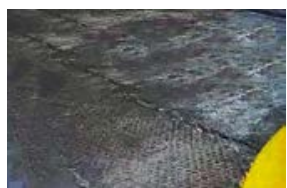

(e)

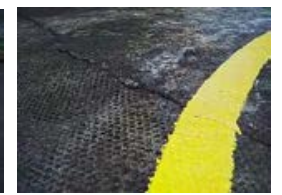

(c)

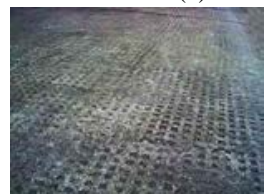

(f)
Figure 9. Simulation process of out of the navigation path. (a) shows the robot run in the center of navigation path, (b) (e) shows the robot out of the navigation path gradually, (f) the robot out of the navigation path already

TABLE IV. Simulation Process of OUT OF the NAVIgATION Path

\begin{tabular}{|c|c|c|c|c|}
\hline Figure & $\begin{array}{c}\text { Otsu } \\
\text { threshold } \\
\left(t_{0}\right)\end{array}$ & $\begin{array}{c}\text { matching } \\
\text { threshold } \\
(\boldsymbol{\mu})\end{array}$ & $\begin{array}{c}\text { gradient } \\
(\boldsymbol{\theta})\end{array}$ & $\begin{array}{c}\text { offset } \\
(\Delta)\end{array}$ \\
\hline Fig.9 (a) & 130 & 0.943 & $-0.491 \pi$ & 7.255 \\
\hline Fig.9 (b) & 135 & 0.955 & $-0.347 \pi$ & 118.961 \\
\hline Fig.9 (c) & 132 & 0.981 & $-0.356 \pi$ & 150.522 \\
\hline Fig.9 (d) & 137 & 0.922 & $-0.403 \pi$ & 158.909 \\
\hline Fig.9 (e) & 134 & 0.639 & $-0.299 \pi$ & 160 \\
\hline Fig.9 (f) & 154 & 0.554 & - & - \\
\hline
\end{tabular}

Table IV shows the Otsu threshold of Fig.9 (f) is more than others too much, there template matching threshold $\mu=0.554$ is less than the matching threshold $=0.85$, then, the 
matching is unsuccessful. So the Condition1, 2, 3 are satisfied, the robot was out of the navigation path already.

\section{B. Applications}

The proposed robust visual navigation method was successfully applied in Shaoshan substation inspection robot in Hunan Province, China. Shaoshan substation is a relatively old substation, there road is narrow, unsmooth and more debris on both sides which in a more complex environment, then it will suffer more interference from outside environment. The stable running of inspection robot in this substation testify the effectiveness of this visual navigation method for complex environments. Fig.10 shown some practical applications of this visual navigation approach to show robot behavior in significant situations.

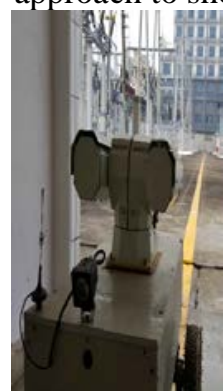

(a)

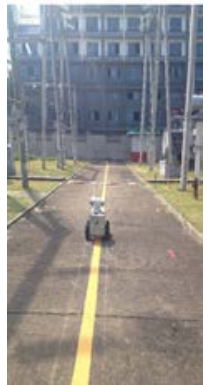

(b)

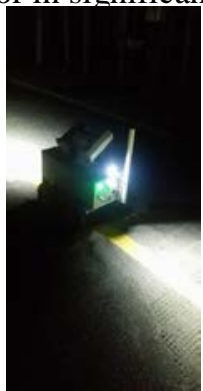

(c)

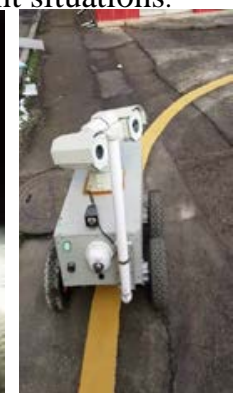

(d)
Figure 10. Application in substation. (a) waiting for inspection tasks. (b) inspecting with the strong sunlight. (c) inspecting in evening. (d) running in the bend

\section{CONCLUSION}

This paper has provided a novel method about robust visual navigation substation inspection robot in complex road environment, with strong anti-interference, the proposed method is simple, accurate and has good stability. Taking advantage of the insensitivity to light changes, shadows and interference in HSV color space, the proposed method can reflect the characteristics information of the navigation path more accurately. Even if out of the navigation path robot can use historical navigation data to automatic return and robot can adjust supplemental lighting intensity that make robot can inspect smoothly in evening. Experiments show that this method works well in practice, effectively solve the navigation problems of vision-based inspection robot in many kinds of complex environment.

\section{ACKNOWLEDGEMENTS}

This work was supported by the national Science Foundation of China (Grant No. 61473049).

\section{REFERENCES}

[1] R. T. Tan, Visibility in bad weather from a single image, in Proceedings of the 26th IEEE Conference on Computer Vision and Pattern Recognition (CVPR'08), Anchorage, Alaska, USA, June2008, 2347-2354.

[2] X. Chen, J. Yang, J. Zhang, and A. Waibel, Automatic detection and recognition of signs from natural scenes, IEEE Transactions on Image Processing, vol.13, no.1, 87-99, 2004.

[3] Vilash Gaidhane, Yogeshv Hote, A computationally efficient approach for template matching-based image registration, Sadhana, Vol. 39, Part 2, 2014, 317-331.

[4] DeSouza G N, Kak A C. Vision for mobile robot navigation: A survey, IEEE Trans on Pattern Analysis and Machine Intelligence, 24(2), 2002, 237-267.

[5] F. Geovani Rodr'guez-Telles, L. Abril Torres-M'endez, A Fast Floor Segmentation Algorithm for Visual-based Robot Navigation, 2013 International Conference on Computer and Robot Vision, 167-173.

[6] Marco L. Della Vedova, Tullio Facchinetti, Antonella Ferrara, Visual interaction for real-time navigation of autonomous mobile robots, 2009 International Conference on Cyber Worlds, 211-218.

[7] Dong Jiwen, Sen Yang, Lu Shouyin, Trajectory Based Substation Patrol Robot's Monocular Visual Navigation, Chinese Journal of Scientific Instrument, 2008,29(4).

[8] Ishita Maiti, Monisha Chakraborty, A New Method for Brain Tumor Segmentation Based on Watershed and Edge Detection Algorithms in HSV Color Model, Conference on Computing \& Communication Systems, 2012, 1-5.

[9] C, Liu, H, Zheng, D, Yu, and X, Xu, A Novel Method of Adaptive Traffic Image Enhancement for Complex Environments, Journal of Sensors, 2015. 